\title{
Mitteilungen der Berliner Gesellschaft für Psychiatrie und Neurologie e.V.
}

\section{Griesinger-Preisträger 2019}

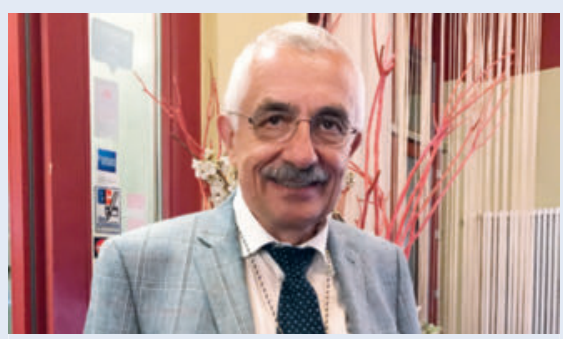

Univ.-Prof. Dr. Erich Schmutzhard, Träger der Berliner Griesinger-Medaille 2019 (Quelle: Dr. Sabrina Thiel, BGPN)

Am 12. Juni 2019 wurde im Rahmen der Mittwochsveranstaltung der BGPN die Griesinger-Medaille an Univ.-Prof. Dr. Erich Schmutzhard verliehen. Er hielt den diesjährigen Griesinger-Vortrag über Neues aus Epidemiologie und Management der Infektionen des Nervensystems.

Erich Schmutzhard wurde in Neumarkt im Mühlkreis in Oberösterreich geboren. Im Jahr 1968 hat er sein Medizinstudium an der Universität Innsbruck begonnen und ist Tirol seither trotz vielfacher Einsätze in fernen Ländern treu geblieben. Nach einem mehrmonatigen USA-Aufenthalt in Cleveland/Ohio schloss er 1974 sein Studium ab. Nach dem Studium war er zunächst 3 Jahre lang als praktischer Arzt am Bezirkskrankenhaus Lienz (Tirol) tätig. Im Jahr 1977 erwarb er ein Diplom für Tropenmedizin und Hygiene in Liverpool. Es folgte eine Beschäftigung für einige Monate in der Neurologie in Innsbruck, danach leitete er 4 Jahre lang das Mnero Hospital in Tansania, um danach in die Neurologie in Innsbruck zurückzukehren, wo er 1987 den Facharzt für Neurologie machte. Seit 1988 leitet er die große neurologische Intensivstation der Universitätsklinik Innsbruck mit heute 18 Betten und ist Universitätsdozent. Seit 2001 hat er eine Professur für neurologische Intensivmedizin an der Universität Innsbruck.

Schmutzhard war Gastdozent bzw. -professor für Tropenneurologie in Bangkok, am
Bernhard-Nocht-Institut in Hamburg und an der Charité Berlin. In Kroatien und Libyen war er Gastprofessor für neurologische Intensivmedizin. Er war und ist des Weiteren in zahlreichen nationalen, europäischen und internationalen wissenschaftlichen Kommissionen zu den Themen Neurologische Intensivmedizin und Tropenneurologie tätig. Bis heute hat er 344 Publikationen in peer-reviewed Journals veröffentlicht. Seine Schwerpunkte sind sowohl die praktische neurologische Intensivmedizin als auch die translationale experimentelle neurologische Intensivmedizin. Auf der anderen Seite hat er bis heute einen lebhaften Kontakt zu Afrika gehalten und sich mit vielen Aspekten der Tropenneurologie, v. a. der Malaria und der Tuberkulose, aber auch zahlreichen anderen Infektionen beschäftigt.

Die BGPN ehrt Schmutzhard für seine Verbindung von engagierter ärztlicher Anteilnahme am einzelnen Patienten und produktiver klinischer und experimenteller Wissenschaft.

\section{VERANSTALTUNGSHINWEISE}

Mittwochsveranstaltungen der

BGPN - 18.00 Uhr

Ort: Hörsaal der Nervenklinik der

Charité Campus Mitte

11.09.2019

Aktuelle Erkenntnisse in der Schizophreniebehandlung;

Prof. Dr. Christoph U. Correll, Charité Berlin

Therapie der Hirnvenenthrombose; PD Dr. med. Florian Masuhr, Bundeswehrkrankenhaus Berlin

20.11.2019

Das Leben und Wirken Wilhelm Griesingers;

Prof. Dr. Michael Seidel, Bielefeld

Genetische Aspekte bei Morbus

Parkinson;
Prof. Dr. Thomas Gasser, Universitätsklinikum Tübingen

11.12.2019

Franz Anton Mesmer als Pionier der Psychotherapie;

Univ.-Prof. Dr. Dr. Dipl.-Psych. Reinhard J. Boerner, Krankenhaus Quakenbrück

Schlafbezogene Atemstörungen und zerebrovaskuläre Erkrankungen;

Dr. Sven Rupprecht, Universitätsklinikum Jena

Herbsttagung Samstag,

26.10.2019 - 10.00 Uhr

Ort: Kaiserin-Friedrich-Haus, Robert-

Koch-Platz 7, 10115 Berlin

Gutachterliche Fragen in der Psychiatrie und Neurologie

Die Rolle des Gutachters: Unterschiede zur Position des Mediziners; Prof. Dr. P. Marx

Fahreignung bei psychiatrischen Erkrankungen; Prof. Dr. Dipl.-Psych. G. Laux

Fahreignung bei neurlogischen Erkrankungen; Prof. Dr. W. Fies

Arbeitsunfähigkeit und Erwerbsunfähigkeit: Nicht können, nicht dürfen, nicht wollen?; Prof. Dr. M. Linden Die Begutachtung des leichten Schädel-Hirn-Traumas; PD Dr. F. Marzinik Teilnahme kostenfrei.

IMPRESSUM

Prof. Dr. Tom Bschor Redaktion: Dr. Anja M. Bauer Berliner Gesellschaft für Psychiatrie und Neurologie e. V.

Schlosspark-Klinik, Abteilung für Psychiatrie

Heubnerweg 2, 14059 Berlin info@bgpn.de,www.bgpn.de 